\title{
Jean-Claude Leclerc
}

\author{
Journaliste, conférencier, journal LE DEVOIR, Montréal \\ (2006) \\ "Religions \\ à l'extrême”
}

Un document produit en version numérique par Jean-Marie Tremblay, bénévole, Professeur sociologie au Cégep de Chicoutimi

Courriel: jean-marie_tremblay@uqac.ca

Dans le cadre de "Les classiques des sciences sociales"

Site web: http://classiques.uqac.ca/

Une bibliothèque fondée et dirigée par Jean-Marie Tremblay, sociologue

Une collection développée en collaboration avec la Bibliothèque

Paul-Émile-Boulet de l'Université du Québec à Chicoutimi

Site web: http://bibliotheque.uqac.ca/ 
Cette édition électronique a été réalisée Jean-Marie Tremblay, bénévole, professeur de soins infirmiers retraitée de l'enseignement au Cégep de Chicoutimi

Courriel: jean-marie_tremblay@uqac.ca

à partir du livre de :

Jean-Claude Leclerc, “Religions à l'extrême”. Un article publié dans la $\boldsymbol{R} \boldsymbol{e}$ vue Notre-Dame, Québec, vol. 104, no 4, avril 2006, pp. 1-14.

L’auteur est journaliste, conférencier, journal LE DEVOIR, Montréal.

[Autorisation formelle accordée par l'auteur, M. Jean-Claude Leclerc et confirmée par Mme Danielle Hébert, directrice de la Revue Notre Dame.]

Sf Courriel : Mme Danielle Hébert, directrice, RND

hebertda@vl.videotron.ca ou rnd@videotron.ca

Site Web: $\quad$ www.revue-rnd.qc.ca

Polices de caractères utilisée :

Pour le texte: Times New Roman, 14 points.

Pour les citations : Times New Roman 12 points.

Pour les notes de bas de page : Times New Roman, 12 points.

Édition électronique réalisée avec le traitement de textes Microsoft Word 2004 pour Macintosh.

Mise en page sur papier format : LETTRE (US letter), 8.5'’ x 11'’)

Édition numérique réalisée le 9 mai 2006 à Chicoutimi, Ville de Saguenay, province de Québec, Canada. 
Un immense merci à la directrice de la Revue Notre Dame de nous assurer de toute sa coopération et de nous autoriser, mardi le 9 mai 2006, conjointement avec l'auteur, M. Jean-Claude Leclerc, la diffusion numérique, dans Les classiques des sciences sociales, de cet excellent article sur les "religions à l'extrême”.

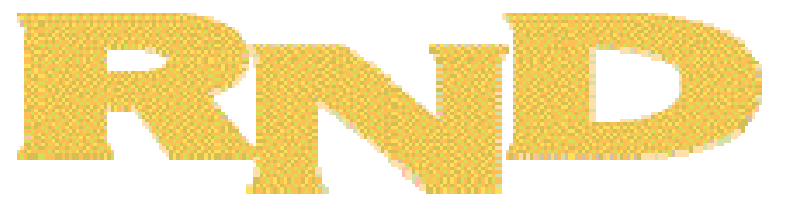

A.

Courriel : $\quad$ Mme Danielle Hébert, directrice, RND hebertda@vl.videotron.ca ou rnd@videotron.ca

Site Web: $\quad$ http://www.revue-rnd.qc.ca 


\section{Jean-Claude Leclerc}

Journaliste, conférencier, journal LE DEVOIR, Montréal

\section{“Religions à l’extrême”}

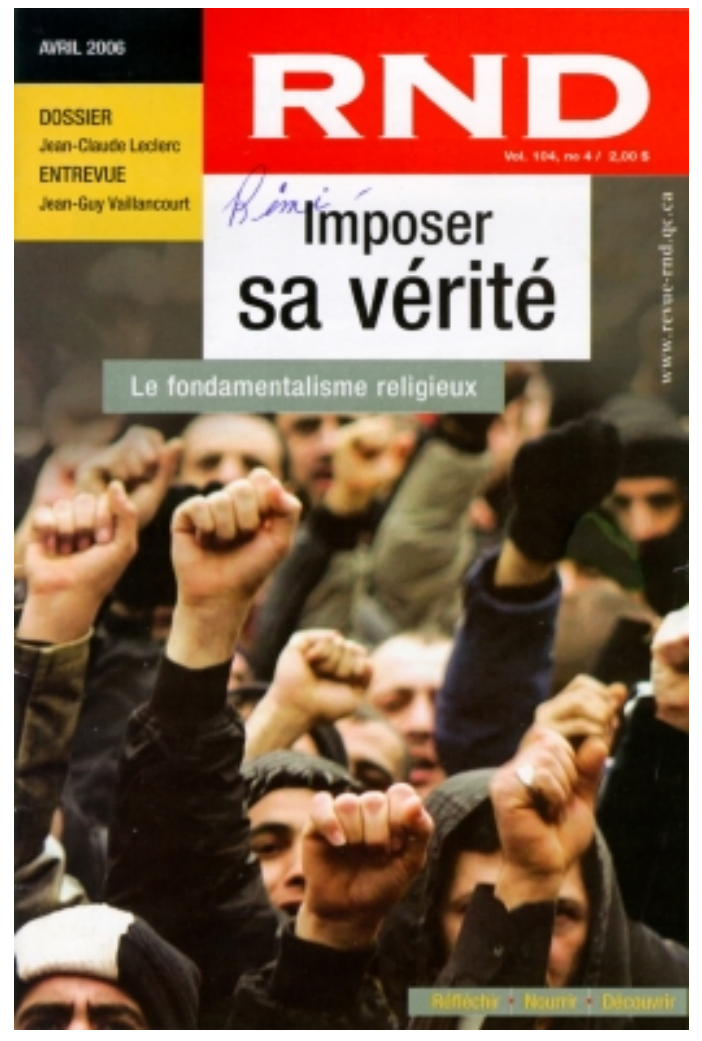

Un article publié dans la Revue Notre-Dame, Québec, vol. 104, no 4, avril 2006, pp. 1-14. 


\section{Table des matières}

$\underline{\text { Introduction }}$

Pour un retour à la « vraie » foi ?

Les croyances ébranlées

Le refus de la modernité

Des défis nous sont posés

\section{Lectures suggérées}




\section{Jean-Claude Leclerc \\ “Religions à l'« extrême »”.}

Un article publié dans la Revue Notre-Dame, Québec, vol. 104, no 4, avril 2006, pp. 1-14.

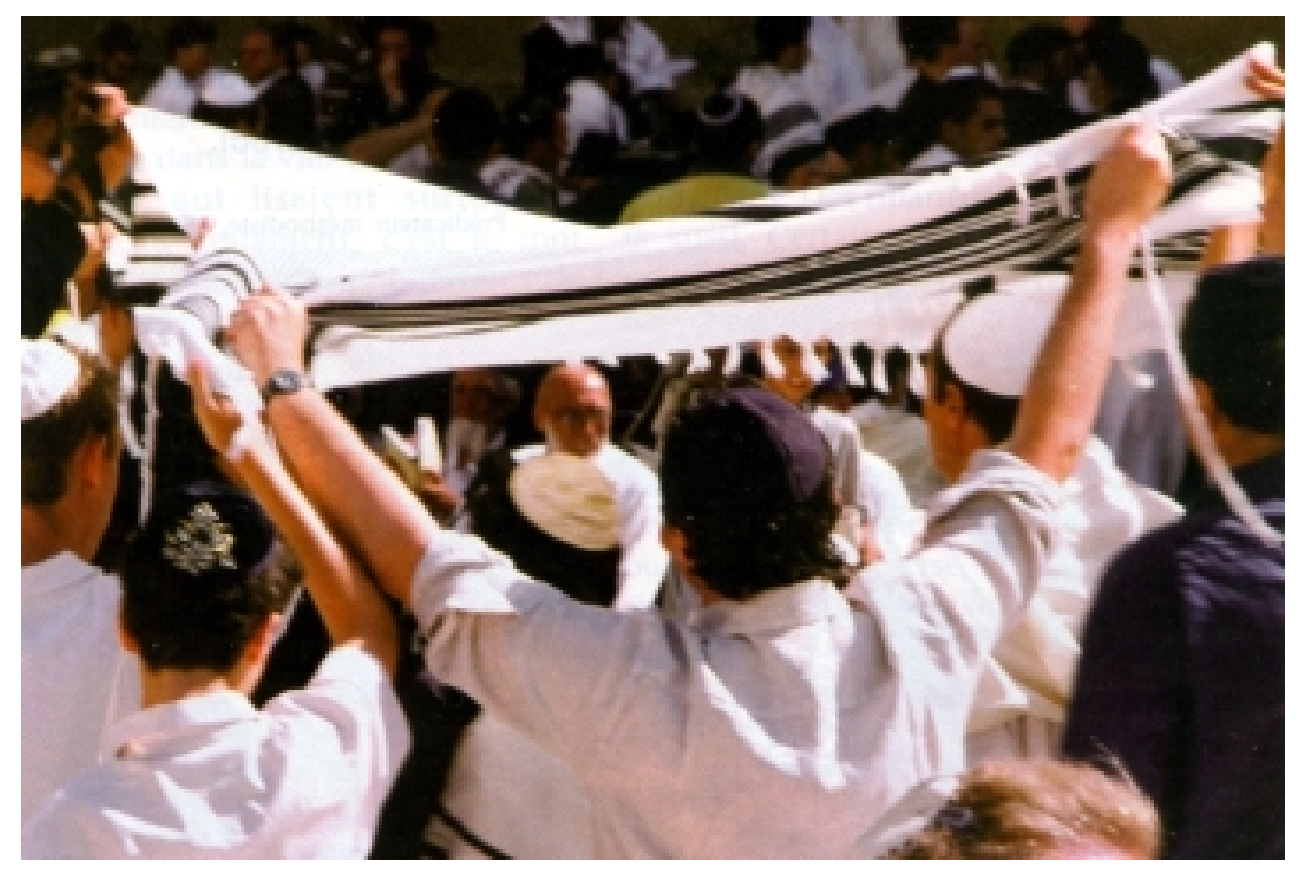

Presque chaque jour, des manifestations religieuses font la manchette de l'actualité. Elles avivent bien souvent les passions et suscitent la controverse. Les gestes extrêmes font peur. Nous redoutons d'en être témoins, et aussi victimes, chez nous. Parallèlement à cette inquiétude, le manque de connaissances peut nous mener à accuser certains groupes d'être responsables de tous les excès. Comment mieux saisir l'extrémisme religieux ? 


\section{Pour un retour à la « vraie » foi ?}

\section{$\underline{\text { Retour à la table des matières }}$}

Dramatisée par les médias, l'actualité politique porte souvent à penser que le fondamentalisme est lié à l'islam. En fait, le phénomène est universel.

Prédicateur méthodiste, 1860, Angleterre

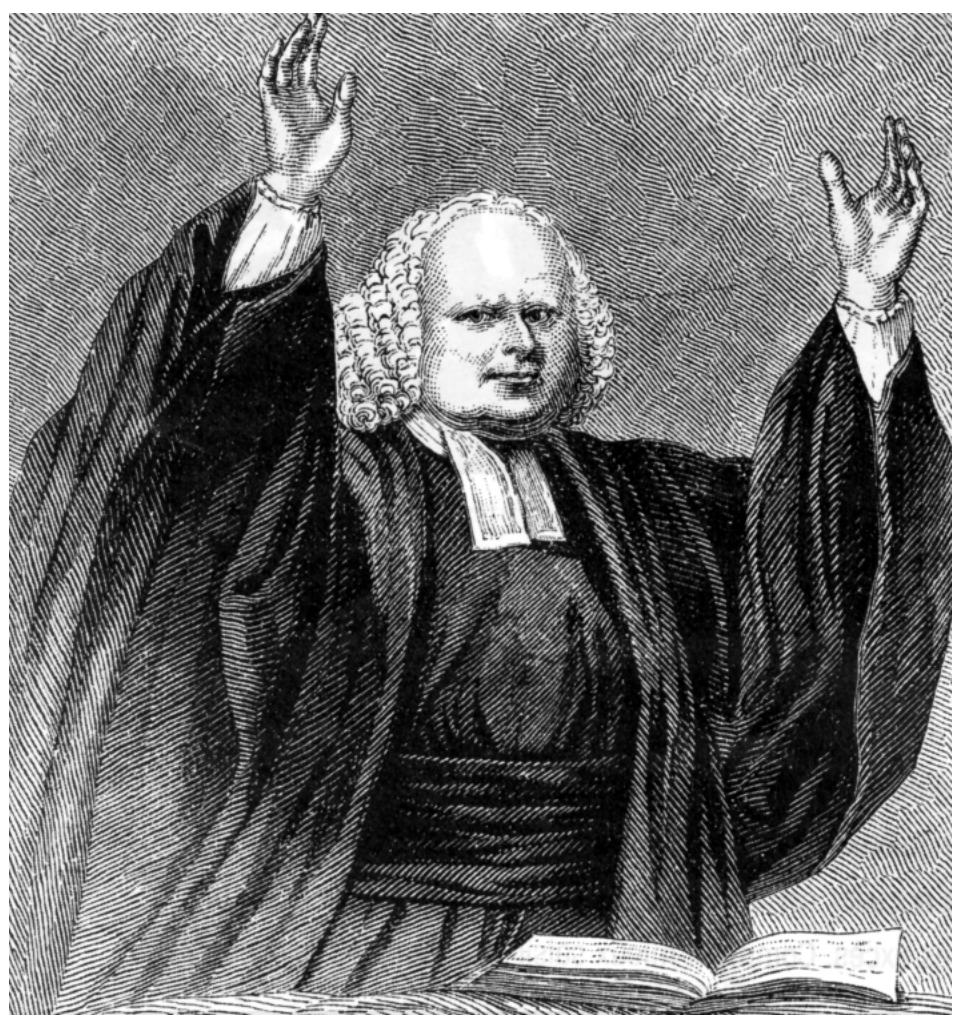

Firstlight (C) Heritage Image Partnership

De nos jours, le fondamentalisme fait peur. Il n'en a pas toujours été ainsi. Au siècle passé, aux États-Unis, ce mot désignait un mouvement au sein du protestantisme. Ses membres pensaient que la $\mathrm{Bi}$ ble, interprétée littéralement, était essentielle à l'authentique foi chrétienne. Il fallait donc y adhérer strictement, dans l'enseignement 
comme dans la vie. Chez les catholiques, qui lisaient surtout le Nouveau Testament, c'est le mot intégrisme qui a désigné le refus de toute évolution de la doctrine.

Les gens qui se replient sur des croyances jugées «fondamentales » sont parfois confondus avec les sectes (qui veut dire " groupe séparé ») ou encore les "nouvelles religions ». Des distinctions s'imposent. Les sectes sont connues pour leur étroite et stricte cohésion autour d'un chef et d'une doctrine. On en trouve quelques-unes au Québec, telle l'Armée de Marie, qui s'est épanouie dans l'Église catholique, et qui a finalement été réprouvée par Rome. On tient aussi pour une secte les Apôtres de l'amour infini. Quant aux nouvelles religions, plusieurs d'entre elles sont aussi présentes au Québec, comme l'Église de scientologie, venue de Californie, ou le Mouvement raëlien, né en France.

Dramatisée par les médias, l'actualité politique porte souvent à penser que le fondamentalisme est lié à l'islam. En fait, le phénomène est universel. On le trouve au sein de plusieurs traditions religieuses. On peut même croire qu'il est de tous les temps. En effet, les querelles sur le sens des écritures sacrées, les modifications aux exercices du culte, le relâchement des exigences morales suscitent, en réaction, des mouvements de « retour à la vraie foi ».

Qu'ils soient nés au Québec ou qu'ils y aient été introduits par des religieux venus d'Europe, différents groupes catholiques ou issus du catholicisme ont quelque chose du fondamentalisme. Les spécialistes préfèrent souvent les désigner comme conservateurs, intégristes ou traditionalistes. Des nuances s'imposent là aussi. Certains groupes sont en marge de l'Église, retirés souvent dans des couvents, comme les disciples de la Contre-Réforme ou de la Fraternité Pie-X, qui se voient comme les dépositaires de l'authentique tradition. D'autres sont apparus avec la crise de l'éducation chrétienne ou avec la controverse, surtout américaine, sur le darwinisme.

Ainsi, l'Association chrétienne de parents-éducateurs du Québec, qui prône l'enseignement à la maison, s'appuie sur une interprétation littérale de la Bible. Pour elle, le livre " est entièrement exempt d'erreurs », qu'il s'agisse de concepts théologiques ou de " précisions géo- 
graphiques et historiques ». Bien que chaque famille membre de l'Association reste "libre d'enseigner les valeurs qu'elle veut à ses enfants », la théorie de l'évolution, par exemple, n'a pas sa faveur : l'univers, croit-elle, a été créé en six jours. Il en va de même pour l'Association de science créationniste du Québec ou encore pour le pendant québécois de Answers in Genesis, groupe américain qui soutient que la Terre n'a que 6000 ans, et non des milliards d'années, comme l'affirment les scientifiques.

Du côté protestant, les principales églises ont souffert du déclin de la natalité, tout comme chez les catholiques, mais aussi de l'exode de milliers d'anglophones. Par contre, des communautés " évangéliques » ont connu, comme aux États-Unis, une croissance importante. En effet, alors que les églises en place -unie, anglicane, presbytérienne et luthérienne - comptaient à peine 158000 membres en 2001, les groupes évangéliques en dénombraient plus de 178000.

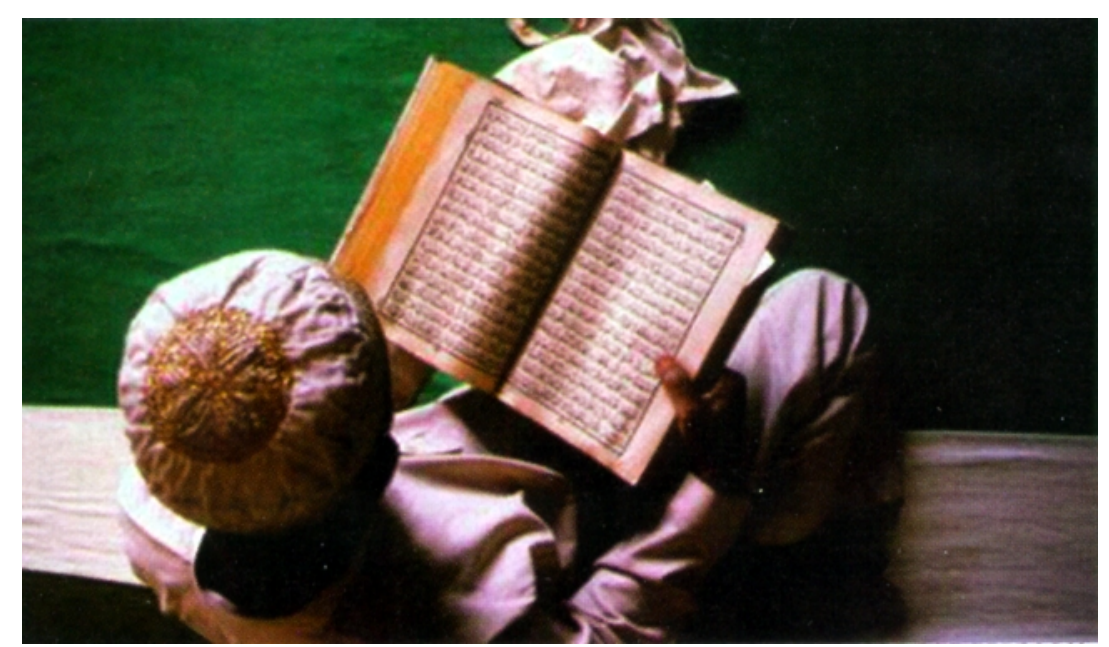

Firstlignt (C) Photodisk

On trouve certes des évangéliques dans les églises traditionnelles. Mais le progrès spectaculaire des nouveaux protestants tient, au Québec, à leur succès auprès de certains immigrants, mais surtout de francophones qui ne pratiquaient plus. La différence d'attrait est nette. Les communautés évangéliques accueillent chaque semaine à l'église près de 64000 participants, alors que les autres sont réduites, le dimanche, à moins de 17 000. Ainsi, en janvier 2001, avec l'aide financière de groupes canadiens et américains, l'Église Nouvelle Vie ouvre ses por- 
tes à Longueuil. Issus d'une vingtaine de religions et de maintes ethnies, ses fidèles sont cependant, en grande majorité, des Québécois d'origine. La plupart sont des jeunes et des gens dans la force de l'âge. On y étudie la Bible, à l'église et à la maison. Des équipes s'adressent spécialement aux alcooliques, aux mères célibataires, aux malades du sida. Plusieurs bands jouent de la musique. Trois chorales sont déjà formées. Le pasteur Claude Houde, son fondateur, est né à Montréal. Formé à l'institut Zion Bible de Providence, au Rhode Island, il a pris de l'expérience dans plusieurs pays. Il résume la préoccupation de son Église en citant la lecture que Jésus a faite du livre d'Isaïe : " je suis venu guérir les cœurs brisés, libérer les captifs, rendre la vue aux aveugles et donner un temps d'espoir. " Le personnel permanent de cette communauté compte une vingtaine de personnes, dont huit ayant des fonctions pastorales, y compris des femmes.

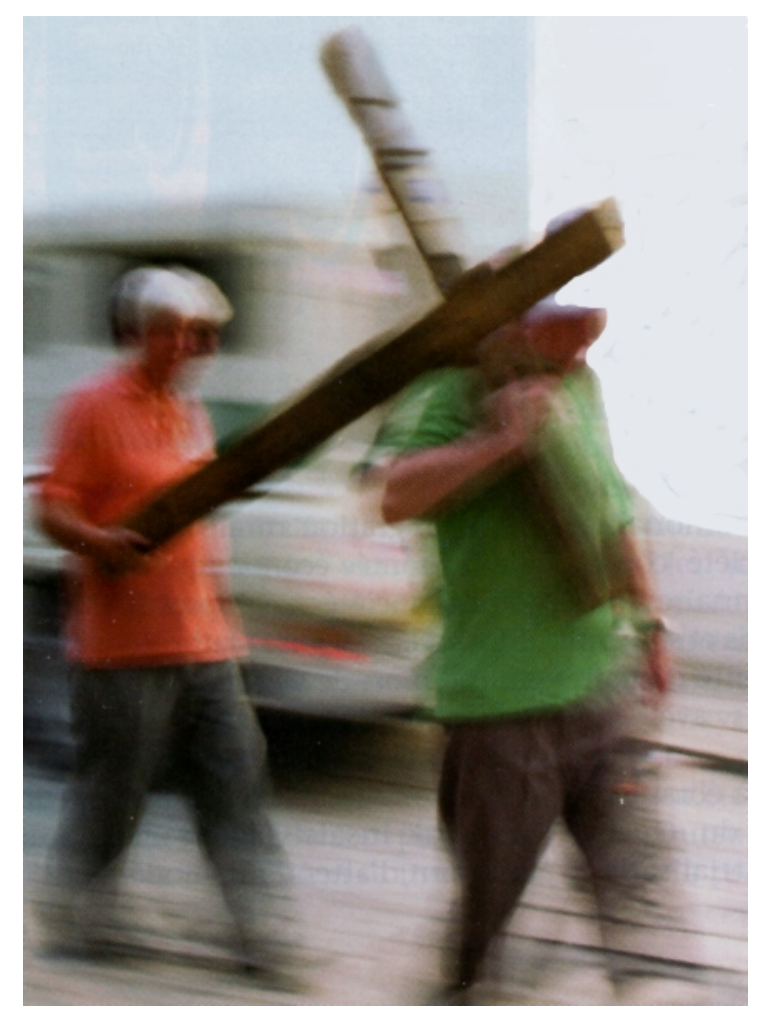

(C) Gettimages / M. Docher

Moins portés à recruter des membres à l'extérieur de leur communauté, d'autres groupes comme les juifs orthodoxes s'en tiennent à des observances strictes. On compte cependant divers courants dans le judaïsme. Ainsi, tenus à l'origine pour des hérétiques par les rabbins, 
les juifs hassidiques vivent un idéal de sainteté joyeuse qui remplit leur existence de tous les jours. Ils n'entendent pas cependant convertir la société environnante.

Il en va autrement des musulmans islamistes, par exemple, qui visent non seulement à se protéger des cultures non religieuses, mais à répandre un mode de vie personnel et social. Ils ne sont pas seuls, il est vrai, à porter des signes extérieurs de leur foi. Mais, un peu comme dans le catholicisme québécois aux siècles passés, leurs prédicateurs soutiennent que toute la société doit vivre selon leur idéal religieux.

Quels qu'ils soient, les groupes fondamentalistes du Québec peuvent y trouver une société qui pourrait aussi bien leur faire bon accueil que les repousser.

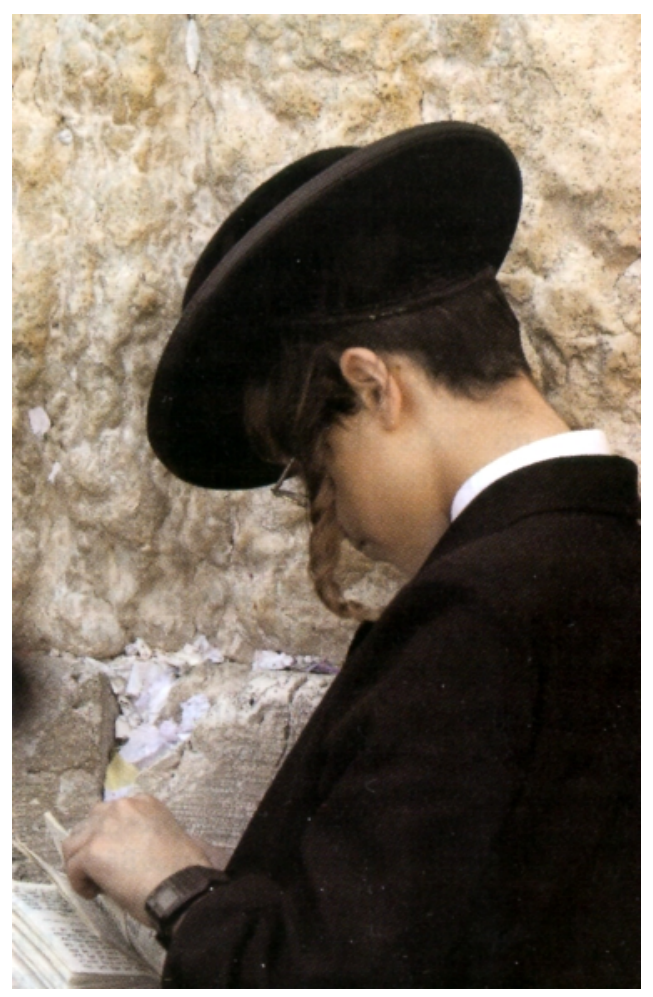

(C) Istokphoto / M. Levit 


\section{Les croyances ébranlées}

$\underline{\text { Retour à la table des matières }}$

Le vide laissé par la chute du catholicisme a créé chez plusieurs un désarroi moral et spirituel. Les besoins fondamentaux, qu'ils soient d'ordre religieux ou philosophique, n'ont pas disparu pour autant.

Contrairement à la Nouvelle-Angleterre, où des colons Il appartenant à des confessions différentes formeront un pays aux croyances diverses, la Nouvelle-France fut fondée sur une seule religion, la foi catholique. Le roi de France y interdisait la présence de juifs et de protestants. Bâtir un nouveau pays et l'Église était alors une seule et même entreprise. Il n'y avait ni raison ni place pour une dissidence religieuse.

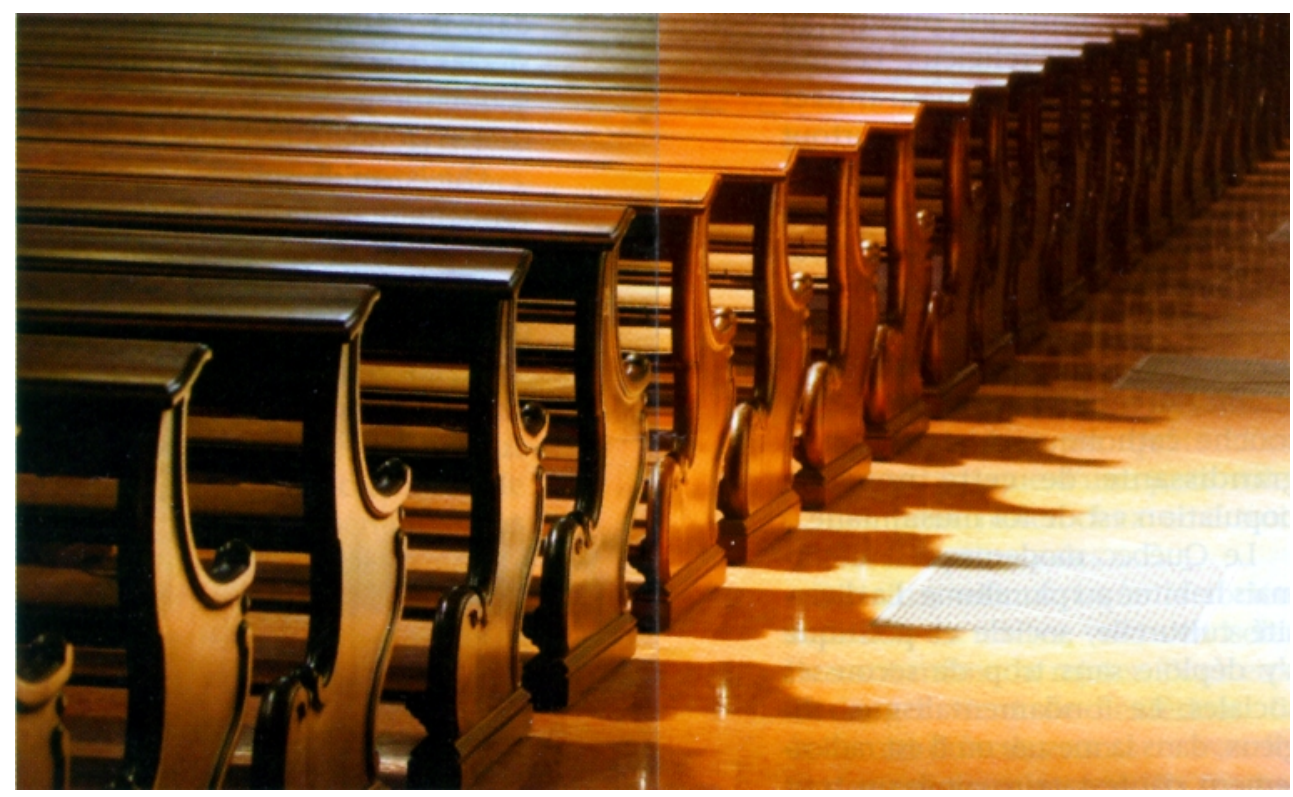

(C) Istokphoto / B. Klumpp

La Conquête britannique, qui y amène un pouvoir anglo-protestant, va consolider, pourtant, une société catholique et française. L'Église remplace la France. Plus tard, des chefs politiques catholiques s'oppo- 
seront au clergé sur des questions politiques. Mais il n'y aura pas de crise au sein de la religion elle-même. À une Église nationaliste correspondait une société confessionnelle. Des missionnaires protestants, parlant français et souvent venus de Suisse, ont tenté d'y faire des conversions, mais avec peu de succès.

Les premiers coups à ébranler la forteresse catholique du Québec sont venus de l'industrialisation, de la migration rurale vers la ville, de la montée économique et sociale d'une société anglo-protestante à Montréal. L'Église catholique s'efforçait alors de soulager la pauvreté, très répandue, puis de combattre, dans la classe ouvrière, la «menace communiste ». Elle ne verra pas qu'un ennemi insaisissable, mais plus puissant, l'attend : la modernité.

En effet, la vie moderne, la consommation, le triomphe de l'individualisme trouvent la religion catholique presque totalement désarmée pour faire face à une mutation culturelle profonde. Les idées sont ébranlées. Les comportements changent. La crise de la foi ne donne pas lieu à des dissidences fondamentalistes, cependant, mais plutôt à une désertion massive des églises. Un même effritement va toucher les confessions protestantes.

Plusieurs tentatives de renaissance catholique verront des groupes revenir aux sources de la foi chrétienne. Groupes de la Bible, Action catholique, communautés de base, mouvement charismatique. Aucune de ces initiatives ne va toutefois entrer en dissidence ouverte avec l'Église ou prétendre fonder une confession nouvelle. La modernité a cependant suscité des mouvements ultra-conservateurs revendiquant une fidélité à la foi traditionnelle.

Ainsi en est-il des Pèlerins de saint Michel. Ils ont été parfois confondus avec les militants du Crédit social (qui souvent portaient aussi un béret blanc). Leur journal Vers Demain a longtemps publié des textes doctrinaux du Vatican. Encore au début de l'an 2000, on s'y faisait un devoir de combattre, selon l'expression d'une dirigeante, la franc-maçonnerie, la haute finance, les mauvaises doctrines et la dépravation des mœurs. Le Crédit social est disparu comme parti politique. Mais les Pèlerins de saint Michel demeurent. 
Comme les mormons et les témoins de Jéhovah, les Pèlerins ont souvent fait du porte-à-porte pour recruter des membres, surtout en milieu rural. Ils se sont également répandus dans la francophonie à l'extérieur du Québec. Mais, sauf à leur siège social de Rougemont, ils ne vivaient pas repliés comme d'autres communautés dans des institutions en marge de la société.

Les Apôtres de l'amour infini, implantés dans un monastère à Saint-Jovite et dans un couvent à Montréal, se sont recrutés également en milieu rural canadien-français et dans les minorités francophones du Canada. Ils ont fait aussi du recrutement dans les Caraïbes, aux États-Unis et même en Europe. Ils ont défrayé l'actualité quand leur chef et ses proches, traduits en justice, ont été libérés, le ministère qui les poursuivait n'ayant pas de preuve à présenter contre eux.

Ces groupes sont restés marginaux et exceptionnels dans le paysage religieux du Québec. Cela tient au fait que, si maints catholiques ont quitté la religion, ce n'est pas parce qu'ils auraient voulu en préserver des articles de foi. Le catholicisme québécois était déjà conservateur et traditionaliste. L'Église aura été désertée par les fidèles avant même d'avoir tenté de s'accommoder avec la société moderne. Les nostalgiques de la religion d'autrefois n'ont pas eu à fonder de groupes fondamentalistes : ils trouvent dans les églises qui subsistent de quoi répondre à leurs besoins.

Chez les nombreux fidèles qui ont tourné le dos à la religion, et chez leurs enfants qui ne l'ont que peu connue, le vide laissé par la chute du catholicisme a créé chez un grand nombre un désarroi moral et spirituel considérable. Les besoins fondamentaux, qu'ils soient d'ordre religieux ou philosophique, n'ont pas disparu pour autant. On ne compte plus les groupes qui ont trouve, parmi ces gens en quête de sens et d'appartenance, des membres de tous âges.

Certains appartiennent à des traditions asiatiques ou à des mouvements de croissance personnelle. D'autres sont plus inquiétants. Ainsi, l'ordre du Temple solaire, secte secrète totalement méconnue au Québec, y fit une sortie spectaculaire, dans une immolation collective meurtrière, qui a semé la stupéfaction. Ce fait est exceptionnel. On peut néanmoins penser qu'au Québec le terrain est propice à l'appari- 
tion de groupes qui savent donner aux gens des règles de vie simples et une communauté où ils se reconnaissent.

Or, il ne manque pas de groupes fondamentalistes pour s'intéresser au Québec. Plusieurs des églises évangélistes des États-Unis sont vigoureusement missionnaires. Aucun coin de la planète ne leur est indifférent. Certaines ont commencé à s'installer au Canada. Des mouvements américains y financent des églises. Une puissante coalition apporte aussi son soutien à des lobbies religieux. Ces organisations ont envahi jusqu'aux campagnes électorales.

Une autre porte est ouverte au fondamentalisme religieux. Les immigrants des prochaines années vont continuer de venir surtout d'Asie et d'Afrique. La plupart voudront s'intégrer en douceur dans leur société d'accueil. Mais les mouvements fondamentalistes y feront aussi du recrutement. Dans le cas du Québec, les immigrants sont encore, au moins pour la moitié, de souche catholique. Mais une part grandissante de cette nouvelle population est de foi musulmane.

Le Québec moderne s'est désormais habitué au pluralisme. La diversité culturelle, sociale et politique s'y déploie sans trop de secousses sociales. Le fondamentalisme religieux, dans la mesure où il est radicalement contestataire, pose un tout autre défi. C'est pourquoi il importe d'en comprendre la naissance et la portée.

\section{Le refus de la modernité}

$\underline{\text { Retour à la table des matières }}$

Diverses causes expliquent le fondamentalisme religieux. Ces mouvements ne proviennent pas d'un zèle soudain pour l'authenticité ou de la seule présence d'une forte personnalité. Des circonstances particulières les font naître.

Bouleversés par des changements dans la société ou au sein de leur religion, les fondamentalistes sont en quête d'un refuge autant que d'une juste voie. 
On peut noter chez eux trois tendances principales :

- Certains, estimant que la société ambiante est nocive, vont s'en isoler. Ils vont même la rejeter. Pour eux, le danger vient de l'extérieur. Ils éviteront donc les contacts avec les autres, religieux ou non. Ils vont se retirer dans des institutions à part, porter des signes et des vêtements marquant leur rupture avec les manières de vivre de leur époque.

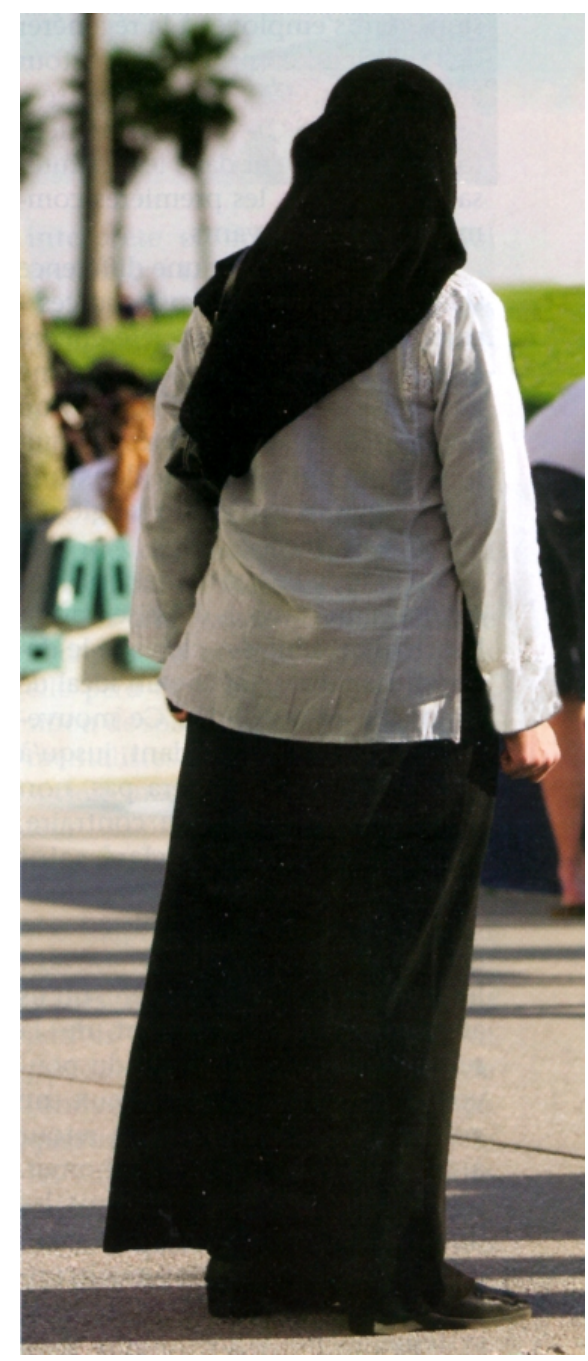

(C) Istockphoto/ M. BLACKBURN

- D'autres, jugeant eux aussi que le monde va à sa perte, vont plutôt tenter de le sauver en l'attirant à leurs croyances. Ils ne pensent pas que seul un "petit nombre » peut accéder à la vérité ou " être sauvé ». ils sont ouverts sur la société. Pour diffuser leur message, ils ont re- 
cours aux grands rassemblements et à des médias de masse. Ils sont actifs à l'échelle de la planète.

- D'autres encore, trouvant que leur institution religieuse en est venue à manquer aux exigences de sa mission, vont s'employer à la régénérer de l'intérieur, en prônant un retour aux sources. Ces sources censées révéler les vrais fondements de leur foi, ils les trouvent dans les écritures sacrées ou dans les premières communautés de croyants.

On doit aussi faire une différence selon que ce " retour aux sources » se produit dans une société encore globalement religieuse (comme autrefois la chrétienté d'Europe, ou de nos jours des sociétés musulmanes ou hindoues) ou dans une société qui a cessé d'être religieuse, comme au Québec.

Au Moyen Âge chrétien, par exemple, un François d'Assise, voyant l'enrichissement des clercs et le désarroi des fidèles, prêche un idéal de simplicité évangélique. Ce mouvement ne va pas, cependant, jusqu'à rejeter l'Église ; il ne sera pas, non plus, rejeté par elle. Au contraire, son fondateur sera déclaré saint par Rome.

On trouve de tels réveils au sein de l'islam contemporain. Devant les problèmes de leur société, qu'ils attribuent au relâchement de la religion et à la corruption du pouvoir, des militants religieux ne voient de salut que dans un retour à la foi du prophète Mahomet. L'ennemi, en somme, ce sont les dirigeants qui s'éloignent du Coran.

Ces islamistes en ont aussi contre l'influence de l'Occident, le système économique dominant, la liberté démocratique, la primauté de l'individu sur la communauté, la tolérance pour la diversité des mœurs. Certaines de leurs critiques sont aussi partagées par d'autres courants religieux. N'a-t-on pas qualifié de fondamentalistes certaines positions du pape Jean-Paul Il ?

En général, dans toutes les religions, les périodes de crise, de changement social et de transformation économique sont propices à un retour radical ou à un attachement visible aux symboles et aux principes. Les changements technologiques qui bouleversent les habitudes culturelles provoquent également des réactions de défense. 
De plus, l'identification étroite d'une religion avec un groupe ou avec un territoire est aussi de nature à causer des crises allant jusqu'à la violence. Ainsi, le nationalisme hindou a provoqué de graves émeutes en Inde. L'identification à la « terre d'Israël » par des juifs ultrareligieux a donné lieu à des actes extrémistes. Le mahatma Gandhi fut tué par un extrémiste hindou, et le premier ministre Yitzhak Rabin par un extrémiste juif.

Quelle que soit leur religion, les fondamentalistes soutiennent que l'homme ne saurait décider librement de ses choix. La vérité ne peut dépendre de débats sociaux ni du vote d'une assemblée. Pour eux, la démocratie est mauvaise car elle repose sur les forces de l'argent ou du nombre : on y fait n'importe quel compromis pour profiter du pouvoir.

Société traditionnelle et religion ne font qu'un. Même quand plusieurs religions cohabitent dans un pays, la vie sociale est fondée sur la religion, elle y trouve son inspiration, ses valeurs et ses règles. La société sécularisée, au contraire, repose sur des valeurs jugées communes qui, sans s'opposer nécessairement à la religion, n'en dépendent pas.

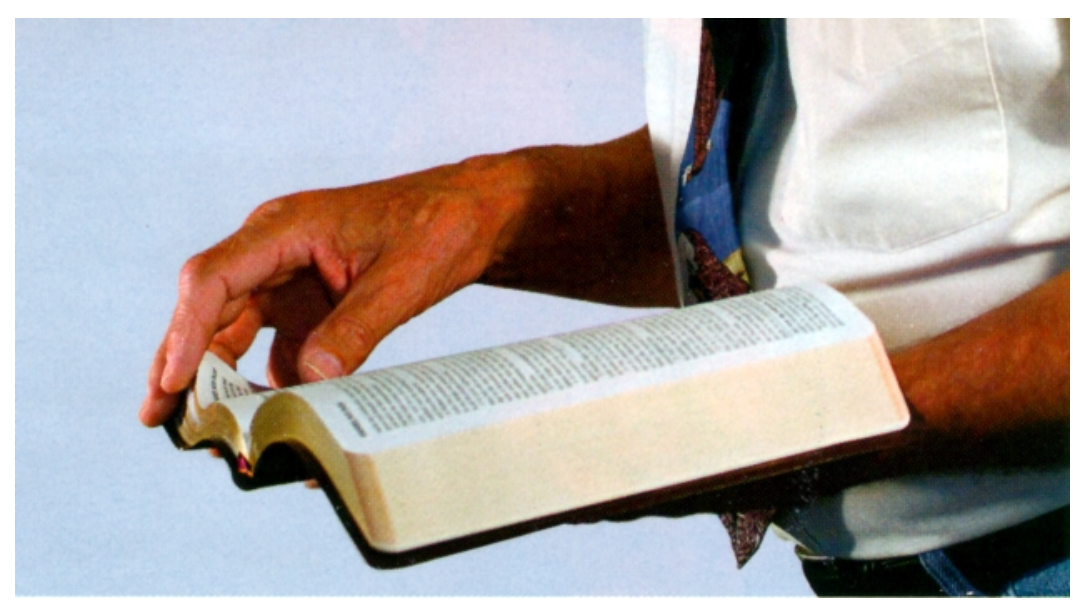

(C) Istockphoto/

À l'ère moderne, les sociétés séculières ou laïques reposent surtout sur des droits humains dits fondamentaux. Les grandes religions s'en accommodent et même les défendent. Il en va autrement des fondamentalistes. Ainsi, l'auteur Louis Schweitzer écrit : «Pour un fonda- 
mentaliste protestant, la notion même des droits de l'homme apparaît presque comme impie. Comme si affirmer ces droits revenait à empiéter sur ceux de Dieu. L'homme ne peut avoir que des devoirs, ceux de la créature devant son Créateur. »

De même, quand Vatican Il réconcilie l'Église catholique avec le pluralisme et les libertés, Mgr Lefebvre déplore le « libéralisme » qui anime les pères du concile. "Le libéralisme en matière de foi et de religion est une doctrine qui prétend émanciper l'homme plus ou moins de Dieu, de sa loi et de sa révélation ; émanciper aussi la société civile de toute dépendance à l'égard de la société religieuse, de l'Église, gardienne, interprète et maîtresse de la loi révélée par Dieu. »

Les fondamentalistes rejettent les compromis doctrinaux que leur religion peut faire en vue de s'ajuster à la modernité ou pour se rapprocher d'autres religions. Ils s'opposeront donc aussi à l'œcuménisme. Pour eux, c'est une erreur que de pactiser avec des croyances qu'encore hier on tenait pour erronées.

Plus une grande religion tente de s'adapter au monde moderne, plus elle perd de son ancienne identité, sinon de son authenticité. Les fondamentalistes qui rejetaient déjà la société moderne, incompatible avec leur vision religieuse des choses, vont aussi rejeter leur propre Église, dans la mesure où celle-ci consent à des adaptations qu'ils tiennent pour des trahisons.

Les fondamentalistes prennent parfois le pouvoir, mais ils sont partout minoritaires. Pourtant, le fondamentalisme, en mettant en question le changement, en signale les enjeux ; il rappelle aussi des valeurs qui risqueraient, autrement, d'être laissées pour compte. Quelles perspectives s'ouvrent à ces communautés au Québec ? Quelle attitude aura-t-on à leur égard ? 


\section{Des défis nous sont posés}

Après avoir appris à cohabiter pacifiquement, catholiques et protestants du Québec vont devoir s'entendre aussi avec de nouvelles communautés. Cela les oblige à remettre à l'ordre du jour la question des religions, de l'héritage culturel qu'ils vont laisser et des valeurs qu'ils veulent maintenir.

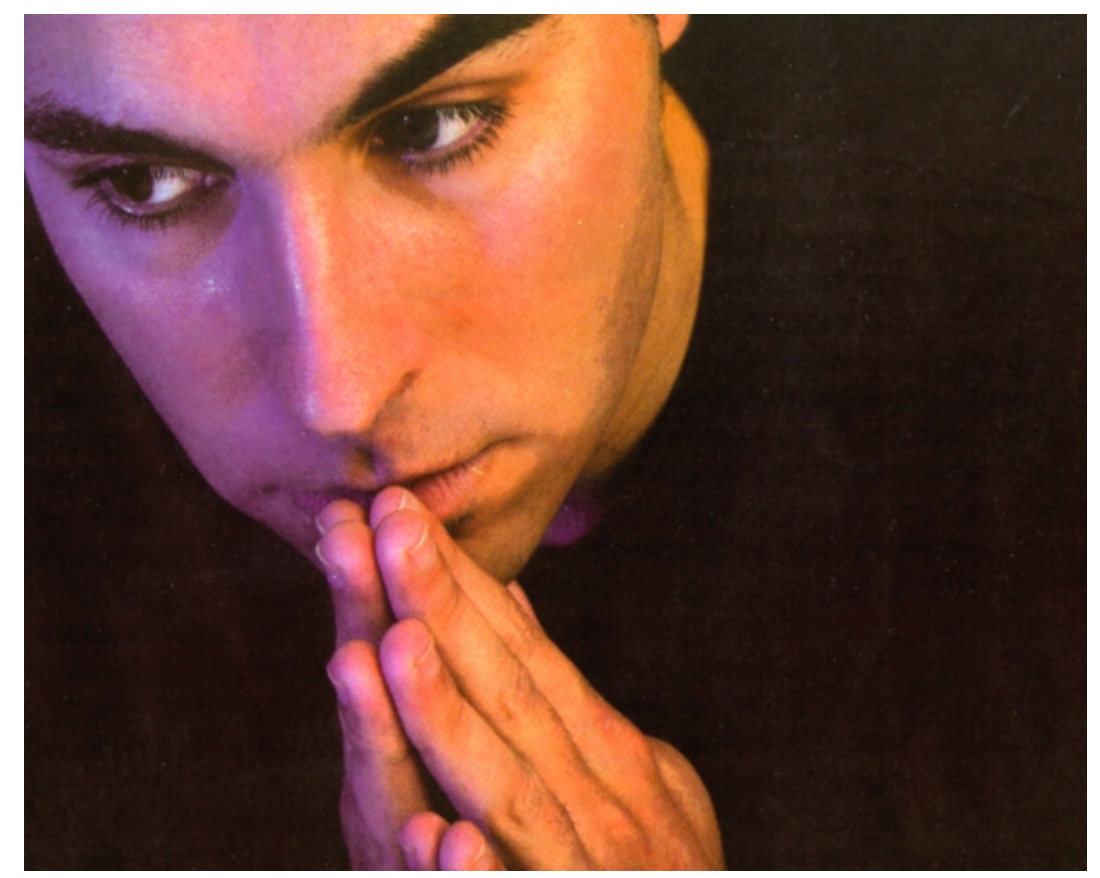

(C) Istockphoto/ E. Serrabassa

Des grandes églises chrétiennes ont cessé de prendre les Écritures saintes au pied de la lettre. Mais en même temps, elles ont perdu leur ascendant sur les gens, même quand ils se disent encore catholiques ou protestants. Elles n'inquiètent plus guère les sociétés dans lesquelles elles vivent. Par contre, avec leur sens de la mission, leur cohésion, 
leur radicalisme moral, les fondamentalistes représentent, eux, une force qui peut déranger.

Les catholiques intégristes ne sont pas jugés capables de poser un défi à la société québécoise. Il en ira peut-être différemment des chrétiens évangéliques ou des musulmans islamistes venus des États-Unis ou de pays musulmans et qui font des conversions parmi les chrétiens qui ne pratiquent plus. Deux secteurs de la société québécoise seront, le cas échéant, interpellés : les églises établies et les milieux prônant la laïcité.

Après avoir appris à cohabiter pacifiquement, catholiques et protestants du Québec vont devoir, en effet, s'entendre aussi avec ces nouvelles communautés. Il en sera de même pour ceux qui excluent toute religion de la vie publique en s'inspirant de la République française, ouvertement hostile aux sectes et au « communautarisme » religieux.

L'incapacité de l'État de répondre aux besoins de certaines populations a permis aux évangéliques et aux islamistes de prendre la relève. Au Québec, ces groupes peuvent, en plus, invoquer la liberté de religion et d'association pour mettre en place leurs propres institutions. Ils le font déjà en éducation. Le recul de l'État providence pourrait leur ouvrir le champ de l'aide sociale. Et l'idée de tribunaux religieux suscite déjà une vive controverse.

Ces croyances qui s'affirment obligent à remettre à l'ordre du jour, dans les écoles comme dans les médias, la question des religions, de l'héritage culturel qu'elles ont laissé, et des valeurs qu'elles veulent maintenir dans la vie privée comme dans la vie publique. Refuser de les entendre ou, pire encore, ne pas tenir compte de leurs différences, c'est repousser ces communautés dans une plus forte marginalité. C'est consacrer en somme le refuge qu'elles offrent aux opprimés et aux oubliés de la société moderne.

Pour le professeur Reginald W. Bibby, spécialiste des religions au Canada, l'Église catholique du Québec pourrait et devrait renouer avec ses fidèles qui ne pratiquent plus mais croient encore, à la condition de représenter elle-même cet « engagement » auquel ils sont encore 
réceptifs. Les églises protestantes elles-mêmes, qui accueillent d'anciens catholiques, devraient, à son avis, former des alliances " créatives et productives » avec les catholiques.

Les grandes religions peuvent se sentir menacées par la montée des communautés fondamentalistes. Ces mouvements leur reprochent en somme d'avoir dérogé aux exigences d'une foi dont elles étaient les gardiennes. Or, une tout autre approche «fondamentale » à l'endroit des religions existe qui est susceptible d'ébranler tant les églises établies que les mouvements fondamentalistes. C'est la recherche des fondements historiques des religions par les spécialistes des vestiges du passé.

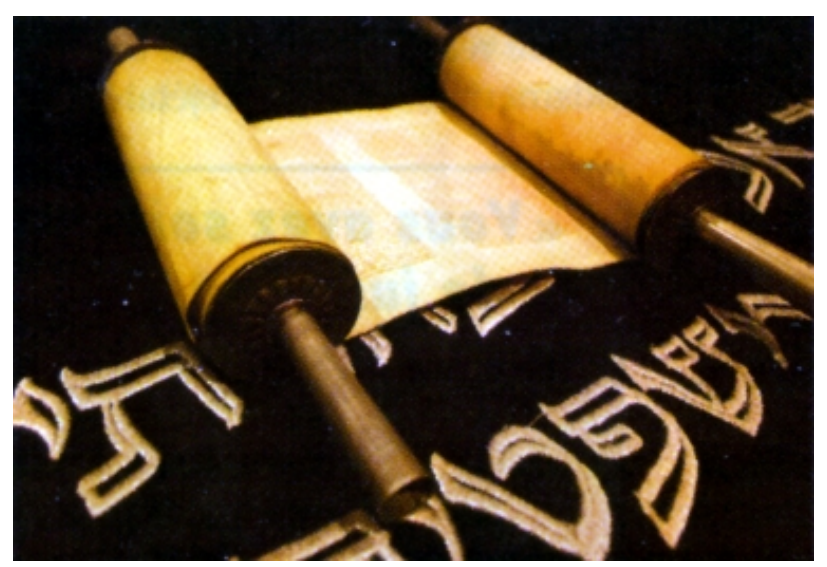

(C) Istockphoto / L. Rosen-Zohar

Des chercheurs, en effet, versés dans les textes sacrés, les coutumes anciennes, les mythes et les croyances d'avant les origines du christianisme, ont commencé à en exhumer les «fondements ». Ils ne s'en tiennent pas à dire qu'on doit parfois lire la Bible de manière symbolique. Ils ont analysé les faits rapportés dans les Évangiles, cherché l'origine des paroles attribuées à jésus. Chez les juifs aussi, des recherches de cette nature ont ébranlé la lecture qu'on faisait traditionnellement des livres de la Bible.

Concernant le christianisme, des chercheurs concluent, par exemple, que Jésus a bel et bien existé, mais qu'il n'a pas fondé de religion ni prétendu être Dieu lui-même. Quand ces interprétations, de plus en plus relayées par les médias, notamment la télévision, vont devenir 
populaires, tout un choc va secouer le christianisme et les cultures qui reposent sur ses dogmes.

Mieux connus dans les pays de langue anglaise et de culture protestante, ces travaux commencent à peine à circuler ailleurs. Ils viennent tout juste d'arriver au Québec. Quel en sera l'impact? On peut prévoir qu'il va en sortir au moins deux types de réactions. D'abord chez les fondamentalistes qui rejetaient la science, ces découvertes historiques vont être également rejetées. On peut aussi penser que bien des croyants traditionnels, ébranlés par le " nettoyage scientifique » de leurs croyances, vont trouver refuge dans des communautés de stricte observance.

Ensuite chez les pratiquants insatisfaits, la réaction sera peut-être de trouver, dans une foi épurée de croyances séculaires, de quoi redonner un sens à une démarche morale ou spirituelle adaptée à leur vie actuelle. Ce courant théologique n'a pas encore soulevé au Québec autant d'intérêt que les nouvelles religions. Seuls quelques savants des sciences religieuses en ont parlé. Mais tôt ou tard une pensée aussi radicalement nouvelle devrait susciter un grand intérêt dans une société en quête d'authenticité.

Jean-Claude Leclerc

Journaliste, Le Devoir.

\section{Lectures suggérées}

$\underline{\text { Retour à la table des matières }}$

Schlegel, Jean-Louis. La loi de Dieu contre la liberté des hommes : intégrismes et fondamentalismes. Paris, Seuil, 2003, 140 p.

HARDY, René. Contrôle social et mutation de la culture religieuse au Québec (1830-1930), Montréal, Boréal, 1999, 267 p.

GIROUX, Gertrude. La spiritualité de Jésus, perdue et retrouvée. Montréal, Carte Blanche, 2002, 199 p. 